\title{
Pazopanib monotherapy in a patient with a malignant granular cell tumor originating from the right orbit: A case report
}

\author{
SACHI MORITA ${ }^{1}$, MARIKO HIRAMATSU ${ }^{2}$, MIHOKO SUGISHITA ${ }^{1}$, BISHAL GYAWALI ${ }^{1}$, TAKASHI SHIBATA ${ }^{1}$, \\ TOMOYA SHIMOKATA ${ }^{1}$, HIROSHI URAKAWA ${ }^{1}$, AYAKO MITSUMA $^{1}$, SUZUKO MORITANI $^{3}$, \\ TOSHINOBU KUBOTA ${ }^{4}$, SHU ICHIHARA ${ }^{3}$ and YUICHI ANDO ${ }^{1}$ \\ ${ }^{1}$ Department of Clinical Oncology and Chemotherapy, Nagoya University Hospital, Nagoya, Aichi 466-8560; \\ ${ }^{2}$ Department of Otorhinolaryngology, Nagoya Graduate School of Medicine, Nagoya, Aichi 466-0027; \\ Departments of ${ }^{3}$ Pathology and ${ }^{4}$ Ophthalmology, National Hospital Organization Nagoya Medical Center, \\ Nagoya, Aichi 460-0008, Japan
}

Received August 2,2014; Accepted April 14, 2015

DOI: $10.3892 / \mathrm{ol} .2015 .3263$

\begin{abstract}
Granular cell tumors are uncommon, usually benign tumors of Schwann cell origin. The malignant variant is extremely rare, representing $<2 \%$ of all granular cell tumors. Therefore, standard systemic chemotherapy for this disease does not exist. The present study reports the case of a 40-year-old female with a malignant granular cell tumor that originally arose in the right orbit and subsequently relapsed. The patient was started on pazopanib monotherapy following treatment with two investigational drugs, a smoothened inhibitor and then a phosphatidylinositol 3-kinase inhibitor, as part of a clinical trial. Although additional radiotherapy for local control was necessary, the lung metastases remained stable during the pazopanib monotherapy, which lasted for 7 months, following which a clinically stable disease state was determined. This case suggests that pazopanib can be a treatment option for the stabilization of disease progression in metastatic malignant granular cell tumor.
\end{abstract}

\section{Introduction}

Granular cell tumors (GCTs) are rare tumors that can occur at various sites in the body, with the most common locations being the skin and subcutaneous tissues (1). GCTs are believed to be Schwann cell derivatives (1) and are listed under the neural tumors in the 2006 World Health Organization (WHO) classification of tumors (2). The majority of GCTs are benign, and malignant cases are scarce, representing $<2 \%$ of all GCTs $(2,3)$. The new WHO classification of tumors of the soft tissue and bone incorporates peripheral nerve sheath tumors, including GCTs, into a category with soft-tissue tumors (4).

Correspondence to: Dr Sachi Morita, Department of Clinical Oncology and Chemotherapy, Nagoya University Hospital, 65 Tsurumai-cho, Showa-ku, Nagoya, Aichi 466-8560, Japan

E-mail: s-kamei@med.nagoya-u.ac.jp

Key words: pazopanib, malignant granular cell tumor, orbit
For patients with unresectable malignant GCTs, the current clinical opinion is to treat the condition in line with the sarcoma-based protocol (5). However, there is little evidence for the use of systemic therapy for this rare malignancy, with only a few published case studies (6-8).

The present study reports a case of metastatic malignant GCT treated with pazopanib monotherapy.

\section{Case report}

In 2011, a 40-year-old female was referred to Nagoya University Hospital (Nagoya, Japan) for treatment of a malignant GCT that originally arose in the right orbit and subsequently relapsed. The primary tumor was initially resected surgically when the patient was aged 38 years, followed by two other resections for local recurrence after a year. The pathological findings of the last resected tumor met five of the six criteria (spindling, vesicular nuclei with large nucleoli, increased mitotic activity, a high nuclear to cytoplasmic ratio and pleomorphism, but no necrosis) proposed by Fanburg-Smith et al (3) to define histological malignancy (Fig. 1). No other chemotherapy or radiotherapy was administered. At the time of presentation to Nagoya University Hospital, the patient exhibited a locally recurrent tumor in the right orbit, and multiple metastases in the pulmonary and cervical lymph nodes. The patient initially received radiotherapy ( $50 \mathrm{~Gy} / 25$ fractions) to the local lesion for pain control, followed by treatment with a smoothened inhibitor and then a phosphatidylinositol 3-kinase inhibitor in the setting of a clinical trial. The primary and metastatic lesions remained stable for 9 months during treatment with the latter agent. When tumor regrowth was diagnosed, pazopanib monotherapy was commenced at a standard oral dose of $800 \mathrm{mg}$ daily, 2 weeks after completing treatment with the last investigational drug. At 2 months after the initiation of pazopanib treatment, computed tomography scans of the chest showed that a number of the pulmonary metastases had partially shrunk (Fig. 2). The major adverse events included grade 2 fatigue and grade 2 hypertension. The hypertension was well controlled using $5 \mathrm{mg}$ amlodipine 
A

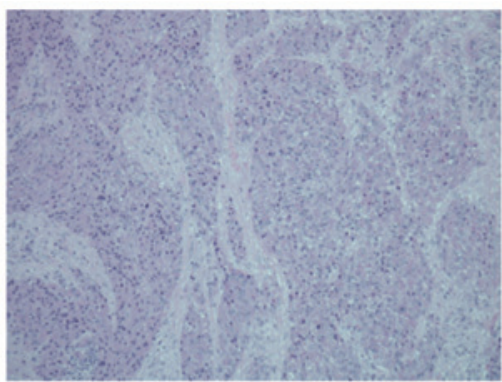

D

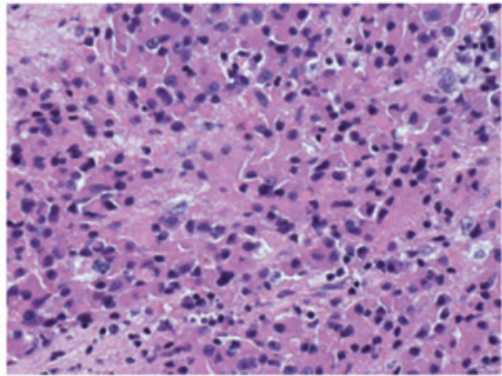

B

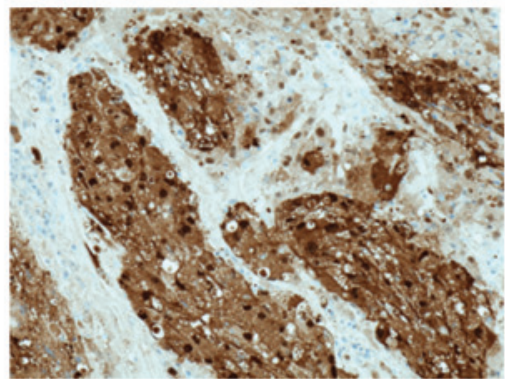

E

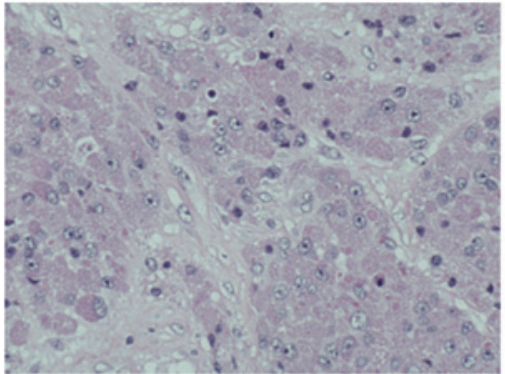

C

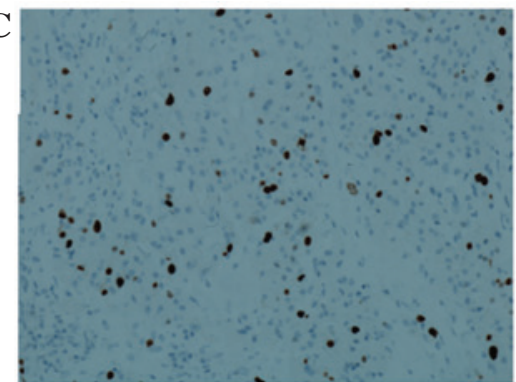

F

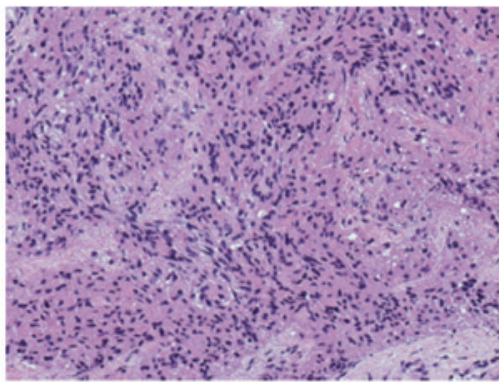

Figure 1. Pathological features of the tumor that (A) consisted of cells with abundant eosinophilic granular cytoplasm [hematoxylin and eosin (H\&E) staining; magnification, x200] and (B) stained positive for S-100 (magnification, x200). (C) The MIB1-index was 9.7\% (magnifcation, x200) and the tumor exhibited (D) a high nuclear to cytoplasmic ratio (H\&E; magnification, $\mathrm{x} 400)$, (E) vesicular nuclei with large nucleoli (H\&E; magnification, $\mathrm{x} 400)$ and (F) spindle cells (H\&E; magnification, $\mathrm{x} 200$ ).

A

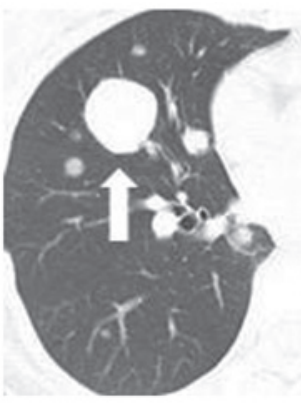

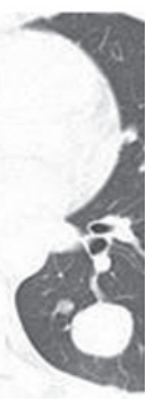

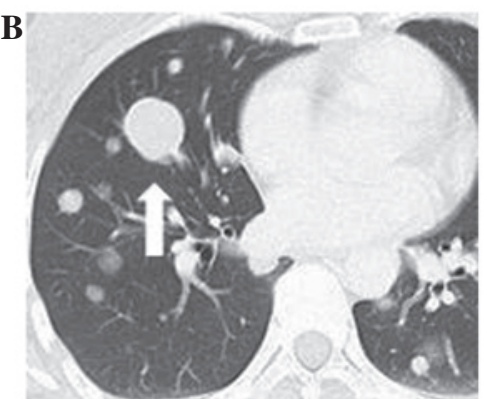

Figure 2. Chest computed tomography scans (A) prior to pazopanib treatment and (B) 2 months after pazopanib treatment began, showing shrinkage of a few of the pulmonary metastases (arrow).

tablets, once a day. Although additional radiotherapy to the local recurrent tumor (34 Gy/17 fractions) and cervical lymph nodes (40 Gy/20 fractions) was considered necessary, the lung metastases remained stable during the pazopanib monotherapy for 7 months, suggesting that pazopanib was effective at least in terms of stabilizing disease progression. Pazopanib therapy was discontinued after 7 months due to fatigue. Next-generation sequencing using the Foundation Medicine T5a platform (Foundation Medicine, Inc. Cambridge, MA, USA) implemented in the clinical trial by Novartis revealed that the tumor from the last surgical resection exhibited a somatic mutation in the additional sex comb-like 1 (ASXL1) gene (1174C>T; Q592*) (9).

This study was approved by the institutional review board of Nagoya University Hospital, and consent was obtained from the patient.

\section{Discussion}

Malignant GCTs are rare tumors. The criteria proposed by Fanburg-Smith et al (3) for diagnosing malignant GCTs is well-known. However, the diagnosis between a benign tumor and a malignant tumor without metastases is difficult. Also, in the present case, the primary tumor behaved in a benign fashion and acquired aggressiveness whenever it locally recurred. Following the last resection of the local recurrence, it metastasized within a short span of only 6 months. For malignant GCT, the only standardized therapy is a local wide excision, and the role of chemotherapy and radiotherapy remains indefinite. To the best of our knowledge, there has been only one reported case of a good response of a malignant GCT to chemotherapy (6). Malignant GCT is believed to be an aggressive chemorefractory disease. Recently, Conley et al (7) reported a case of metastatic malignant GCT in which pazopanib monotherapy achieved a response; this was similar to the experience recorded for pazopanib monotherapy in the present study. Pazopanib is an oral, small-molecule inhibitor of vascular endothelial growth factor receptor-1, -2 and -3 , platelet-derived growth factor receptor- $\alpha$ and $-\beta$, and $c-k i t$, which has shown single-agent activity in patients with advanced soft-tissue sarcomas $(10,11)$. Pazopanib therapy was used in the present study as the phase 
III study of pazopanib in advanced soft-tissue sarcoma included one patient with a malignant GCT who experienced stable disease and tumor shrinkage $(8,11)$. To this date, three patients with malignant GCT who achieved clinical benefits with pazopanib monotherapy, including the present case, have been recorded. This is a noteworthy finding for the treatment of this rare tumor.

The present patient had been enrolled in investigational drugs studies and had undergone next-generation sequencing. The tumor exhibited a somatic mutation in the ASXL1 gene (1174C>T; Q592*). The ASXL1 gene is one of the human homologues of the Drosophila Asx gene, which is located at human chromosome 20q11.21. ASXL1 protein is involved in transcriptional activation and repression in a context-dependent manner (9). The initial study on human ASXL1 mutation was performed in 2009, in patients with myeloid malignancies (12). Now, gene amplification and truncation mutations of ASXL1 are known to exist in various types of cancer (9). Although $A S X L$ is worthy of note as a new target of cancer therapy, it is necessary to clarify the clinical significance of $A S X L$ gene alternation. This is the first report to discover the presence of the ASXL1 mutation in a patient with a malignant GCT.

In conclusion, the results of the present study together with two previous cases suggest that pazopanib may be a safe, effective and reasonable treatment option for GCT. This finding should be investigated and validated, preferably by clinical trials or given the rarity of this disease, by other case studies.

\section{References}

1. Fisher ER and Wechsler H: Granular cell myoblastoma - a misnomer. Electron microscopic and histochemical evidence concerning its Schwann cell derivation and nature (granular cell schwannoma). Cancer 15: 936-954, 1962.
2. Argenyi ZB: Granular cell tumor. In: WHO Classification of Tumors. Pathology and Genetics of Tumors of the Skin. LeBoit PE, Burg G, Weedon D and Sarasin A (eds). IARC press, Lyon, pp274-275, 2006.

3. Fanburg-Smith JC, Meis-Kindblom JM, Fante R, et al: Malignant granular cell tumor of soft tissue: diagnostic criteria and clinicopathologic correlation. Am J Clin Pathol 22: 779-794, 1998.

4. Fanburg-Smith JC: Malignant granular cell tumor. In: WHO Classification of Tumors of Soft tissue and Bone. Fletcher CDM, Bridge JA, Hogendoorn PCW and Mertens F (eds). IARC press, Lyon, pp190, 2013.

5. Peter S and Olencki T: Unusual cutaneous malignancies. In: Textbook of Uncommon Cancer. Raghavan D, Blanke CD Johnson DH, Moots PL, Reaman GH, Rose PG et al (eds). John Wiley \& Sons, Inc. New Jersey, p679, 2012.

6. Kanat O, Yalcinkaya U, Skbunar T, Hurt E, Evrensel T and Manavoglu O: Gemcitabine plus paclitaxel may be a promising chemotherapy regimen for metastatic granular cell tumor. Clin Oncol (R Coll Radiol) 20: 93-96, 2008.

7. Conley AP, Koplin S, Caracciollo JT, et al: Dramatic response to pazopanib in a patient with metastatic malignant granular cell tumor. J Clin Oncol 32: e107-e110, 2014.

8. Evaluation and Licensing Division, Pharmaceutical and Food Safety Bureau, Ministry of Health, Labour and Welfare: Report on the deliberation results. Votrient tablets $200 \mathrm{mg}$. September 11: $1-132,2012$

9. Katoh M: Functional and cancer genomics of ASXL family members. Br J Cancer 109: 299-306, 2013.

10. Sleijfer S, Ray-Coquard I, Papai Z, et al: Pazopanib, a multikinase angiogenesis inhibitor, in patients with relapsed or refractory advanced soft tissue sarcoma: a phase II study from the European organisation for research and treatment of cancer-soft tissue and bone sarcoma group (EORTC study 62043). J Clin Oncol 27: 3126-3132, 2009

11. Van der Graaf WT, Blay JY, Chawla SP, et al: Pazopanib for metastatic soft-tissue sarcoma (PALETTE): a randomised, double-blind, placebo-controlled phase 3 trial. Lancet 379: 1879-1886, 2012.

12. Gelsi-Boyer V, Trouplin V, Adélaïde J, et al: Mutations of polycomb-associated gene $A S X L$ lin myelodysplastic syndromes and chronic myelomonocytic leukaemia. Br J Haematol 145: 788-800, 2009. 